\title{
Interplay of entrainment and rheology in snow avalanches: a numerical study
}

\author{
Dieter ISSLER, ${ }^{1}$ Manuel PASTOR PÉREZ ${ }^{2}$ \\ ${ }^{1}$ Norges Geotekniske Institutt and International Centre for Geohazards, PO Box 3930, Ullevål Stadion, \\ NO-0806 Oslo, Norway \\ E-mail: di@ngi.no \\ ${ }^{2}$ ETS de Ingenieros de Caminos, Universidad Politécnica de Madrid, Ciudad Universitaria, ES-28040 Madrid, Spain
}

\begin{abstract}
A one-dimensional evolution equation for the slope-normal velocity profile of a streamwise uniform avalanche over an entrainable bed is derived. The boundary conditions are no slip at the bed, a stress-free surface and constant bed shear stress equal to the shear strength of the snow cover. The resulting equation is solved numerically by means of finite differences on a regular grid with a superposed fine grid near the erosion front that is adjusted at each time-step. The first exploratory simulations yield realistic entrainment rates and show that the entrainment rate tends towards a constant value while the flow depth and the velocity increase linearly with time for all investigated rheologies. It is shown that there indeed exists a rheology-independent asymptotic solution to the equation of motion of an entraining slab if the bottom friction is equal to the bed shear strength; the asymptotic acceleration is found to be half the downslope gravitational acceleration. The model can easily be extended to general path profiles, non-uniform flows and variable snow properties.
\end{abstract}

\section{INTRODUCTION}

Since quantitative measurements of the mass balance of snow avalanches have been reported (Issler and others, 1996; Sovilla and others, 2001; Vallet and others, 2001; Sovilla, 2004), modellers have renewed their efforts to describe the important but poorly understood process of entrainment. Different erosion mechanisms have been hypothesized (Gauer and Issler, 2004), and for some of these (frontal ploughing, ripping of chunks corresponding to an entire snowpack layer and continuous abrasion along the flow bottom) there seems to be conclusive experimental evidence from the deposit structure or data from profiling radar (Gubler and Hiller, 1984; Issler, 2003; Sovilla, 2004). To date, the potential of laboratory chutes for detailed studies of erosion and entrainment mechanisms with diverse bed and flow materials under controlled conditions has barely been tapped (Barbolini and others, 2005).

Due to the lack of conclusive experimental knowledge, mathematical models of bed entrainment have mostly been heuristic (see Eglit and Demidov, 2005, for a comparison of a large variety of entrainment models developed for different phenomena), typically of the form

$$
q_{\mathrm{e}} \propto\left(\sigma_{\mathrm{b}}-\tau_{\mathrm{c}}\right)^{a} \quad \text { or } \quad q_{\mathrm{e}} \propto\left(u-u_{\mathrm{c}}\right)^{b},
$$

where $a$ and $b$ are empirical exponents, $\sigma_{\mathrm{b}}$ is the bed shear stress and $\tau_{\mathrm{C}}$ and $u_{\mathrm{c}}$ are the threshold, or critical, bed shear strength and velocity, respectively. More physical approaches rooted in fluid dynamics were proposed by the Moscow school, either treating the flow/bed interface as a shock front (Briukhanov and others, 1967; Grigorian and Ostroumov, 1977) or postulating an analogy with shear-induced mixing at the interface between different fluids (Eglit, 1983). In the case of (frictional, dry) granular flows on a bed of the same material, Boutreux and others (1998) clarified the role played by the slope angle. Their work was extended and applied to snow avalanches by Naaim and others (2004), who may have been the first to recognize the close connection between the shear rate at the bed/flow interface (governed by the rheology of the flowing material) and the entrainment rate.

D. Issler and T. Jóhannesson (unpublished information) further explored the connections between flow rheology, bed properties, erosion of bed particles and their entrainment into the flow, assuming constant flow depth and a perfectly brittle bed material that is characterized by its shear strength, $\tau_{\mathrm{C}}$, and is entrained along the flow bottom. With these simplifications, they found analytical solutions for quasistationary flow of a Bingham fluid and realistic erosion rates for parameter values typical of snow avalanches. However, extension to non-stationary situations requires some approximations that are difficult to control a priori, and analytic solutions for the velocity and shear-stress profiles under entrainment may not exist for more complicated rheologies.

Our aim here is therefore to create a tool with which to find suitable approximations to the velocity profiles of entraining flows with nonlinear rheology, or to directly obtain parameterizations of the erosion rate as a function of the depth-averaged flow velocity, $\bar{u}_{x}$, and the flow depth, $h$. We formulate and implement a one-dimensional numerical model for the time evolution of the velocity and shear-stress profiles, the erosion rate and the flow depth that can, in principle, be applied to any rheology of the form

$$
\hat{\tau}=f(\dot{\gamma}, \hat{\sigma})
$$

where $\hat{\tau} \equiv \sigma_{x z} / \rho$ and $\hat{\sigma} \equiv \sigma_{z z} / \rho$ are the specific shear and normal stress, respectively, and $\rho$ is the density. The measure of shear, $\dot{\gamma}$, reduces to the shear-rate gradient, $\partial_{z} u_{x}$, in our idealized situation.

\section{EVOLUTION EQUATION FOR THE VELOCITY PROFILE}

For the sake of simplicity, we make several assumptions, which can easily be relaxed or modified later: (1) We treat the avalanche as infinitely long in the $x$-direction along a slope 


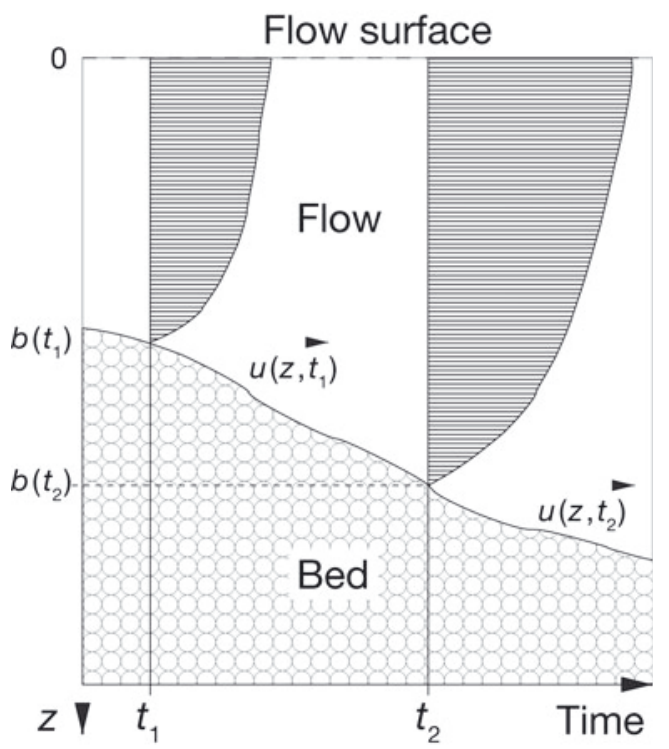

Fig. 1. Schematic $t-z$ diagram, showing the erosion front advancing in time and the choice of coordinate system. Note that the $x$ direction is not shown. The slope of the curve, $b(t)$, is the (nonmaterial) erosion speed, $w_{\mathrm{e}}(t)$. There is no mass flux in the $z$ direction even though mass flows across the moving interface.

inclined at a constant angle, $\theta$. All variables thus depend only on the slope-perpendicular coordinate, $z$, and the time, $t$ (Fig. 1). (2) The snow cover and the avalanche are assumed to have the same density, $\rho$. (3) Entrainment stops as soon as the entire snow cover is eroded away. (4) The combined depth of avalanche and snow cover remains constant, i.e. there is no mass redistribution along the body of the avalanche. As a consequence, a particle in the avalanche always moves parallel to the ground and the interface between flow and bed is also parallel to the ground.

Mass balance is trivially satisfied in this set-up, and the momentum balance in the $z$-direction yields $\hat{\sigma}=-z g_{\perp}$ if we assume the surface at $z=0$ to be stress-free and $\left(g_{\|}, g_{\perp}\right) \equiv(g \sin \theta, g \cos \theta)$ is the gravitational acceleration vector. With $u(z, t)$ the velocity in the $x$-direction, we write the balance of $x$-momentum as

$$
\partial_{t} u=g_{\|}+\partial_{z} \hat{\tau} .
$$

The shear stress is to be expressed as a function of the shear rate, $\partial_{z} u$ (cf. Equation (2)), so Equation (3) in general is a nonlinear partial differential equation of diffusion type for $u$. Note that $\hat{\tau}<0$ for $u>0$ in our coordinate system.

We assume no slip at the erosion front, i.e. $u(b, t)=0$. To completely specify this problem with a time-dependent computational domain, the erosion speed, $w_{\mathrm{e}}=\dot{b}(t)$, must be determined. For bed materials that are perfectly brittle (implying that they fail instantaneously and without significant strain once the stress reaches a critical value, $-\tau_{\mathrm{C}}$ ) Issler and Jóhannesson (unpublished information) argued that there is a fundamental feedback mechanism that keeps the bed shear stress, $\tau_{\mathrm{b}}$, at the value $-\tau_{\mathrm{c}}$ as long as entrainment is possible. If $\left|\tau_{\mathrm{b}}\right|$ were smaller than $\tau_{\mathrm{c}}$, erosion would cease, so the shear rate and $\left|\tau_{b}\right|$ would increase until erosion resumes. If $\left|\tau_{\mathrm{b}}\right|>\tau_{\mathrm{c}}$, however, the erosion rate would grow and the acceleration of more eroded bed material would reduce $\left|\tau_{b}\right|$. We assume that snow can indeed be modelled as a perfectly brittle material to a sufficient degree of accuracy, given the rapid loading induced by the arrival of the avalanche and the substantial shear stresses exerted by the flow, which are of the order of $h \rho g_{\|} \approx 1-3 \mathrm{kPa}$.

Equation (2) and the boundary condition $\hat{\tau}=-\hat{\tau}_{\mathrm{C}}$ imply that the shear rate at the erosion front is constant in time:

$$
\dot{\gamma}(b(t), t)=f^{-1}\left(\hat{\tau}=-\hat{\tau}_{\mathrm{c}}, \hat{\sigma}\right) \equiv \dot{\gamma}_{\mathrm{c}} .
$$

The progression of the erosion front is driven by the need to maintain the stress boundary condition while the recently eroded material is being accelerated. At time $t+\mathrm{d} t$, the velocity of a particle that was eroded at time $t$ is, according to Equation (3) and to first order in $\mathrm{d} t$,

$$
\begin{aligned}
& u(b(t), t+\mathrm{d} t)=u(b(t), t)+\partial_{t} u(b(t), t) \mathrm{d} t \\
& =0+\left[g_{\|}+\partial_{z} \hat{\tau}(\dot{\gamma}(b(t), t), \hat{\sigma}(b(t), t))\right] \mathrm{d} t \\
& =\left[g_{\|}+\left.\frac{\delta \hat{\tau}}{\delta \dot{\gamma}} \partial_{z} \dot{\gamma}\right|_{\dot{\gamma}=\dot{\gamma}_{c}}+\left.\left.\frac{\delta \hat{\tau}}{\delta \hat{\sigma}}\right|_{\dot{\gamma}_{c}} \partial_{z} \hat{\sigma}(b(t), t)\right|_{z=b(t)}\right] \mathrm{d} t
\end{aligned}
$$

while the erosion front is now located at

$$
b(t+\mathrm{d} t) \approx b(t)+w_{\mathrm{e}}(t) \mathrm{d} t .
$$

The shear rate at the front thus evaluates to

$$
\begin{aligned}
& |\dot{\gamma}(b(t+\mathrm{d} t), t+\mathrm{d} t)| \approx \frac{u(b(t), t+\mathrm{d} t)-0}{b(t+\mathrm{d} t)-b(t)}
\end{aligned}
$$

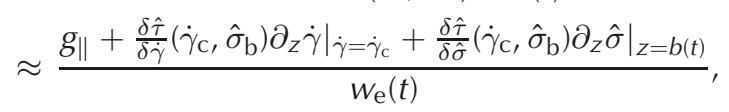

where $\hat{\sigma}_{\mathrm{b}} \equiv \hat{\sigma}(b(t), t)$. As this must be equal to $\dot{\gamma}_{\mathrm{c}}$, we find the propagation speed of the erosion front in the $z$-direction:

$$
w_{\mathrm{e}}(t)=\frac{g_{\|}+\frac{\delta \hat{\tau}}{\delta \dot{\gamma}}\left(\dot{\gamma}_{\mathrm{c}}, \hat{\sigma}_{\mathrm{b}}\right) \partial_{z} \dot{\gamma}(b, t)+\frac{\delta \hat{\tau}}{\delta \hat{\sigma}}\left(\dot{\gamma}_{\mathrm{c}}, \hat{\sigma}_{\mathrm{b}}\right) g_{\perp}}{\dot{\gamma}_{\mathrm{c}}} .
$$

Note that $\dot{\gamma}_{c}$ is time-dependent if the shear stress depends on the normal stress (e.g. due to Coulomb friction). As an example, apply the NIS (Norem-Irgens-Schieldrop) model (Norem and others, 1989),

$$
\begin{aligned}
& \hat{\sigma}=\hat{\sigma}_{\mathrm{e}}-\nu^{\prime} \dot{\gamma}^{2}, \\
& \hat{\tau}=-\hat{\tau}_{\mathrm{y}}+\mu \hat{\sigma}_{\mathrm{e}}-\nu \dot{\gamma}^{2},
\end{aligned}
$$

to Equation (8); here $\mu$ is the dry-friction coefficient, $\hat{\tau}_{y}$ the specific yield strength, $\hat{\sigma}_{\mathrm{e}}$ the specific effective normal stress transmitted by frictional grain contacts, and $\nu, \nu^{\prime}$ are the coefficients of the dispersive stresses $\left(\mathrm{m}^{2}\right)$. The erosion speed becomes

$w_{\mathrm{e}}=\sqrt{\frac{\nu-\mu \nu^{\prime}}{\hat{\tau}_{\mathrm{c}}-\hat{\tau}_{\mathrm{y}}-\mu g_{\perp} h}}\left[g_{\|}-\mu g_{\perp}-2\left(\nu-\mu \nu^{\prime}\right) \dot{\gamma}_{\mathrm{c}} \partial_{z} \dot{\gamma}(b, t)\right]$.

\section{NUMERICAL IMPLEMENTATION AND SIMULATION RESULTS}

Much of the simplicity of Equation (3) is due to the absence of advection in the $z$-direction - a feature that the numerical scheme should reflect. At the same time, there is a moving (non-material) boundary at $z=b(t)$ where increased precision is required because the gradient of the shear rate must be computed at the erosion front (cf. Equation (8)). In order to accommodate both requirements, we combine a coarse regular fixed grid of mesh length $\Delta z_{\mathrm{C}}$ with a fine grid of $N+1$ equidistant nodes a distance $\Delta z_{\mathrm{f}}=\Delta z_{\mathrm{c}} / N$ apart in a finite-difference scheme. At the beginning of each timestep, the fine mesh is translated so that its end coincides 


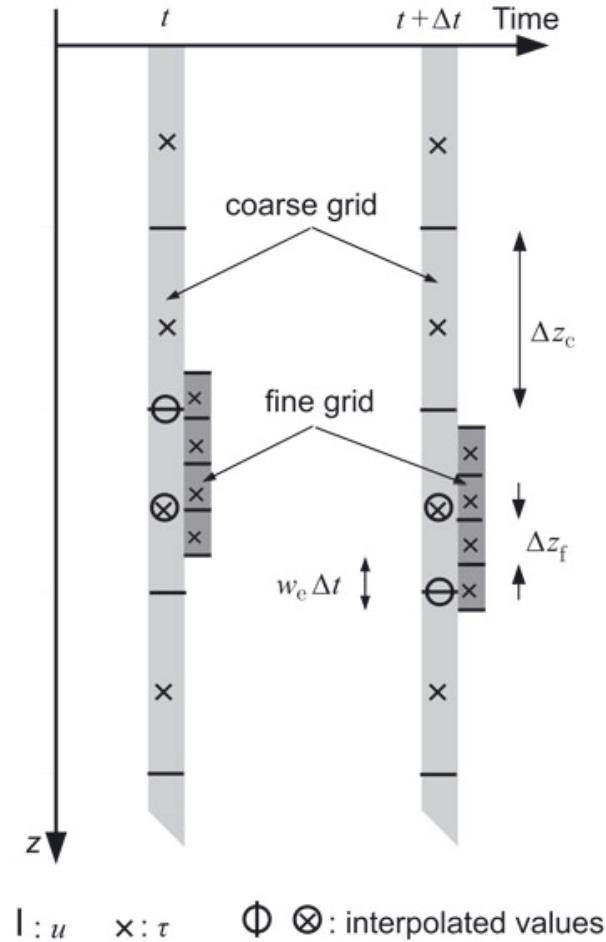

Fig. 2. Schematic representation of the coarse grid and the superposed fine grid, which is shifted to the new position of the erosion front at the beginning of each time-step.

with the present location of the erosion front, and $u, \hat{\sigma}$ and $\hat{\tau}$ are interpolated to the new node positions (see Fig. 2 ). The field values at the coarse-grid node within the fine grid are also interpolated; as the erosion front progresses, successive coarse-grid nodes are activated.

We use staggered grids, with the velocity, $u$, located at the nodes and the stresses, $\hat{\sigma}, \hat{\tau}$, at the cell centres. The $z$-derivatives are approximated by central differences, and quadratic extrapolation is used for $\partial_{z} \dot{\gamma}(b(t))$ so that the scheme is second-order accurate in space. Time-stepping is by simple forward differencing and thus first-order accurate. The time-step is determined from two criteria: (1) the eroded depth per time-step must be less than the cell width of the fine grid; (2) disturbances of the velocity profile, which are damped in the exact solution due to the diffusive nature of the differential equation, must not be amplified in the numerical approximation. The second criterion often imposes very short time-steps, but computation times are nevertheless short (a few seconds) on a current personal computer.

If the erosion rate is a little too large due to small numerical errors, the specific shear stress will drop slightly below $\hat{\tau}_{\mathrm{C}}$, and erosion ceases at once. In order to prevent spurious fluctuations of this kind, the erosion rate is set to $98-99 \%$ of its calculated value.

Another numerical problem, visible in Figure $3 \mathrm{~b}$ and e, arises from the displacement of the fine grid relative to the coarse one: as the tail node of the fine grid is shifted past a node of the coarse grid (which then gets activated), the velocity and shear stress gradients change a little, but they do so abruptly. The disturbance propagates to the erosion front and causes the erosion rate to be overestimated briefly. A more precise interpolation scheme is called for, but the error is negligible for all practical matters.
Table 1. Initial conditions and material properties of the simulations shown in Figure 3

\begin{tabular}{lcc}
\hline Parameter & Newtonian fluid & Bagnoldian fluid \\
\hline Slope angle, $\theta$ & \multicolumn{3}{c}{$30^{\circ}$} \\
Density, $\rho$ & $300 \mathrm{~kg} \mathrm{~m}^{-3}$ \\
Erodible snow depth, $D_{0}$ & $14.0 \mathrm{~m}$ & $15.0 \mathrm{~m}$ \\
Initial flow height, $h_{0}$ & $1.037 \mathrm{~m}$ & $1.05 \mathrm{~m}$ \\
Initial velocity, $\bar{u}_{0}$ & $3.8 \mathrm{~m} \mathrm{~s}^{-1}$ & 0 \\
Snow cover strength, $\tau_{\mathrm{C}}$ & $500 \mathrm{~Pa}$ & $1450 \mathrm{~Pa}$ \\
Rheological exponent, $q$ & 1 & 2 \\
Viscosity, $\eta$ & $0.10 \mathrm{~m}^{2} \mathrm{~s}^{-1}$ & - \\
Dispersive coefficient, $\nu$ & - & $0.005 \mathrm{~m}{ }^{2}$ \\
Dispersive coefficient, $\nu^{\prime}$ & - & $($ arbitrary) \\
Coarse mesh width, $\Delta z_{\mathrm{C}}$ & $0.1 \mathrm{~m}$ & $0.1 \mathrm{~m}$ \\
Fine mesh width, $\Delta z_{\mathrm{f}}$ & $0.02 \mathrm{~m}$ & $0.01 \mathrm{~m}$ \\
& & \\
\hline
\end{tabular}

The code was validated by verifying that non-entraining flows with various rheologies converge to the known analytical solutions for the terminal velocity and stationary velocity profile. In addition, the simulated time evolution of the velocity profile of a non-entraining laminar Newtonian flow matches the theoretical solution obtained by Fourier expansion.

Two exploratory simulations with the new model (Table 1; Fig. 3) assume Newtonian and Bagnoldian rheologies, respectively. The latter is obtained from Equations (9) and (10) as a special case by letting $\hat{\tau}_{y}, \mu \rightarrow 0$. Both simulations deliberately allow unrealistic flow height growth in order to highlight the asymptotic behaviour of the model. If the finite avalanche length and mass redistribution along the flow direction were accounted for, realistic erosion depths of 0.5-2 $\mathrm{m}$ and lower velocities and flow heights would result. While the Newtonian flow was started with an initial velocity and eroded a relatively weak snow cover from the beginning, the snow cover strength for the Bagnoldian case was only slightly below the equilibrium bed shear stress of the nonentraining flow. Erosion therefore began only when the flow had nearly reached terminal velocity, the acceleration had become small and the specific bed shear stress approached the equilibrium value, $h_{0} g_{\|}$. However, after the threshold was passed, the growing flow height made more gravitational force available for entraining snow and accelerating the flow.

The long-term behaviours of both flows exhibit striking similarities, in that both the acceleration and the erosion speed tend towards constant values and the velocity profiles are almost linear except near the surface, where they resemble somewhat the equilibrium profiles of nonentraining flows of the same rheology. Accordingly, the shear-stress profiles are only weakly curved near the surface, but $\partial_{z} \tau$ differs strongly from the stationary value, $\rho g_{\|}=$ $1500 \mathrm{~Pa} \mathrm{~m}^{-1}$. The observed behaviour is a consequence of the crucial postulate that the bed shear stress equal the bed shear strength. This is made clearer in the following section.

\section{ASYMPTOTIC SOLUTIONS FOR SLAB MODELS WITH ENTRAINMENT}

The results obtained through the numerical solutions suggest there exists an asymptotic solution to the equation of motion of an entraining mass point on an inclined plane with constant entrainment rate and linearly growing flow depth 

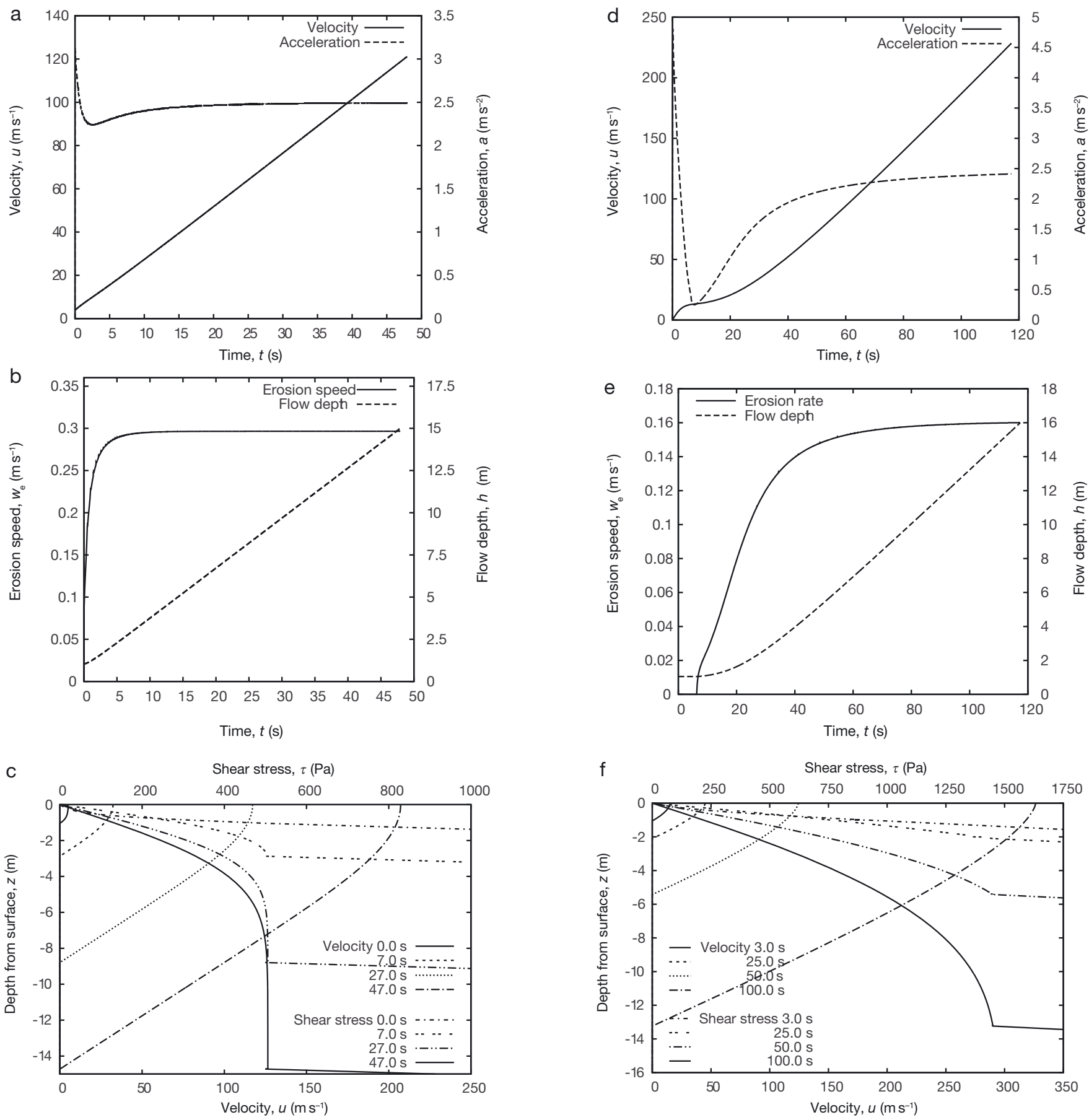

Fig. 3. Results of numerical simulations for the case of Newtonian (left column) and Bagnoldian (right column) fluids in laminar flow. The initial conditions and material properties are summarized in Table 1. The rows show the evolution of, respectively, velocity and acceleration, erosion speed and flow height, and velocity and shear-stress profiles. Note that the Bagnoldian fluid meets the erosion criterion only after $\sim 6 \mathrm{~s}$ when it has almost attained its terminal velocity without erosion of $\sim 17 \mathrm{~m} \mathrm{~s}^{-1}$. The profiles differ strongly from the corresponding equilibrium profiles of stationary non-entraining flows with the same rheology.

and velocity under the shear-stress boundary condition, $\hat{\tau}_{\mathrm{b}}=\hat{\tau}_{\mathrm{c}}$. We therefore set

$$
w_{\mathrm{e}} \rightarrow w_{\infty}=\text { const }, \quad \bar{u} \rightarrow \bar{a}_{\infty} t, \quad h \rightarrow w_{\infty} t
$$

in the equation of motion (overlined quantities are depthaveraged)

$$
\frac{\mathrm{d} \bar{u}}{\mathrm{~d} t}(t)=g_{\|}-\frac{\hat{\tau}_{\mathrm{c}}}{h(t)}-\frac{w_{\mathrm{e}}(t) \bar{u}(t)}{h(t)}
$$

and obtain

$$
\bar{a}_{\infty}=g_{\|}-\frac{\hat{\tau}_{C}}{w_{\infty} t}-\bar{a}_{\infty} .
$$

One immediately sees that $\bar{a} \rightarrow g_{\|} / 2$ as $t \rightarrow \infty$ and the bed shear stress, $\hat{\tau}$, becomes insignificant compared to the gravitational traction, $h g_{\|}$. This conclusion is borne out by the numerical simulations with Newtonian and Bagnoldian rheologies (Fig. 3).

The most notable features of this solution are (1) its independence of the rheology, (2) its apparent stability and (3) the fact that asymptotically one half of the momentum input from gravity goes into accelerating the bulk of the flow and the other half into bringing the eroded mass up to the average flow velocity. 
As mentioned above, some (temporary) model assumptions are unrealistic for snow avalanches, and hence so is this asymptotic solution with ever-increasing velocity and flow height. Interestingly, however, Gauer and others (2010) pointed out that available experimental data on maximum velocity of avalanches, $u_{\max }$, plotted against the drop height, $H$, indicate the relationship $u_{\max } \propto(g H)^{1 / 2}$, which is obtained if the effective friction coefficient is approximately constant, but not if it grows significantly with velocity. The boundary condition on the shear stress we have adopted in this work, $\hat{\tau}_{\mathrm{b}}=\hat{\tau}_{\mathrm{c}}$, gives an effective friction coefficient that is independent of the velocity, but diminishes as the inverse of the growing flow depth. However, entrainment of eroded snow at an ever-increasing speed gives rise to a resistive acceleration, $-a_{\infty} \propto u^{2} \propto g h \propto \hat{\sigma}$, that mimics dry friction as long as the slope angle is constant and there is enough erodible snow. The dynamics of entrainment thus offers a possible explanation for the observed growth of $u_{\max }$ with $H$ that can be tested further against experimental data.

\section{CONCLUSIONS AND OUTLOOK}

The numerical model described here has proved to be a very useful tool to continue the work of Issler and Jóhannesson (unpublished information) beyond the restrictive assumptions needed to obtain analytical solutions. In particular, the asymptotic solution hinted at by the numerical simulations highlights the decisive dynamical role played by the postulated feedback mechanism that locks the bed shear stress at the value of the shear strength of the snow cover, and by the assumptions concerning the redistribution of entrained snow. We anticipate that future detailed studies of the shape evolution of the velocity and shear-stress profiles will give useful guidance in the development of approximate entrainment models based on the self-consistent approach of Issler and Jóhannesson (unpublished information). It is also conceivable that further asymptotic solutions can be found with different assumptions regarding redistribution of entrained snow.

The numerical model also has potential for future development in its own right. Some of the next steps we plan to implement are: (1) improved interpolation methods to reduce disturbances as the fine grid leaves a node of the coarse grid behind; (2) extension to a slab model on an arbitrary path profile, with the slab length growing as snow is entrained; (3) snow strength varying along the path and with depth in the snow cover; (4) the ideas leading to Equation (8) can and should also be incorporated in more advanced numerical models in two dimensions ( $x$ and $z$ ). However, a more refined formulation of the momentum balance will be needed because the assumptions of vanishing $u_{z}$ and independence of $x$ are no longer valid.

Last but not least, there is urgent need for innovative experimental studies of the fundamental entrainment mechanisms. Particularly, our central assumption of perfectly brittle failure needs to be confronted with controlled experiments under realistic conditions. If it holds to some degree in snow, the conditions for its validity in other eroding gravity mass flows are a question of great practical interest. While the experimental challenge is substantial, the pioneering study of Barbolini and others (2005) indicates a promising approach.

\section{ACKNOWLEDGEMENTS}

D.I. gratefully acknowledges a fellowship from the NILS Mobility Project (ABEL Extraordinary Chair action) and the hospitality extended by the Laboratorio de Geotecnia of CEDEX in Madrid, Spain. Completion of this work was financed by the EU Framework Programme 7 project SafeLand and by the Norwegian Water Resources and Energy Directorate through the research project Snow Avalanches. We thank two anonymous referees for careful reading and constructive criticism. This is contribution No. 349 from the International Centre for Geohazards.

\section{REFERENCES}

Barbolini, M., A. Biancardi, F. Cappabianca, L. Natale and M. Pagliardi. 2005. Laboratory study of erosion processes in snow avalanches. Cold Reg. Sci. Technol., 43(1-2), 1-9.

Boutreux, T., E. Raphaël and P.-G. de Gennes. 1998. Surface flows of granular materials: a modified picture for thick avalanches. Phys. Rev. E, 58(4), 4692-4700.

Briukhanov, A.V. and 6 others. 1967. On some new approaches to the dynamics of snow avalanches. In Oura, H., ed., Physics of snow and ice. Sapporo, Hokkaido University. Institute of Low Temperature Science, 1223-1241.

Eglit, E.M. 1983. Some mathematical models of snow avalanches. In Shahinpoor, M., ed. Advances in the mechanics and the flow of granular materials. Vol. 2. Houston, TX, Gulf Publ. Co. ClausthalZellerfeld, 577-588. (Trans. Tech. Publ.)

Eglit, M.E. and K.S. Demidov. 2005. Mathematical modeling of snow entrainment in avalanche motion. Cold Reg. Sci. Technol., 43(1-2), 10-23.

Gauer, P. and D. Issler. 2004. Possible erosion mechanisms in snow avalanches. Ann. Glaciol., 38, 384-392.

Gauer, P., K. Kronholm, K. Lied, K. Kristensen and S. Bakkehøi. 2010. Can we learn more from the data underlying the statistical $\alpha-\beta$ model with respect to the dynamical behavior of avalanches? Cold Reg. Sci. Technol., 62(1), 42-54.

Grigorian, S.S. and A.V. Ostroumov. 1977. Matematicheskaya model sklonovih processov lavinnogo tipa [The mathematical model for slope processes of avalanche type]. Moscow, Moscow State University. Institute for Mechanics (Scientific Report 1955). [In Russian.]

Gubler, H. and M. Hiller. 1984. The use of microwave FMCW radar in snow and avalanche research. Cold Reg. Sci. Technol., 9(2), 109-119.

Issler, D. 2003. Experimental information on the dynamics of drysnow avalanches. In Hutter, K. and N. Kirchner, eds. Dynamic response of granular and porous materials under large and catastrophic deformations. Berlin, Springer, 109-160.

Issler, D., P. Gauer, M. Schaer and S. Keller. 1996. Staublawinenereignisse im Winter 1995: Seewis (GR), Adelboden (BE) und Col du Pillon (VD). Eidg. Inst. Schnee- Lawinenforsch. Interner Ber. 694.

Naaim, M., F. Naaim-Bouvet, T. Faug and A. Bouchet. 2004. Dense snow avalanche modeling: flow, erosion, deposition and obstacle effects. Cold Reg. Sci. Technol., 39(2-3), 193-204.

Norem, H., F. Irgens and B. Schieldrop. 1989. Simulation of snowavalanche flow in run-out zones. Ann. Glaciol., 13, 218-225.

Sovilla, B. 2004. Field experiments and numerical modelling of mass entrainment and deposition processes in snow avalanches. (PhD thesis, ETH Zürich.)

Sovilla, B., F. Sommavilla and A. Tomaselli. 2001. Measurements of mass balance in dense snow avalanche events. Ann. Glaciol., 32, 230-236.

Vallet, J., U. Gruber and F. Dufour. 2001. Photogrammetric avalanche volume measurements at Vallée de la Sionne, Switzerland. Ann. Glaciol., 32, 141-146. 